\title{
TOTAL CHROMATIC NUMBER OF GRAPHS OF HIGH DEGREE, II
}

\author{
H. P. YAP and K. H. CHEW \\ (Received 30 January 1990; revised 31 October 1990)
}

Communicated by L. Caccetta

\begin{abstract}
We prove Theorem 1: suppose $G$ is a simple graph of order $n$ having $\Delta(G)=n-k$ where $k \geq 5$ and $n \geq \max (13,3 k-3)$. If $G$ contains an independent set of $k-3$ vertices, then the TCC (Total Colouring Conjecture) is true. Applying Theorem 1, we also prove that the TCC is true for any simple graph $G$ of order $n$ having $\Delta(G)=n-5$. The latter result together with some earlier results confirm that the TCC is true for all simple graphs whose maximum degree is at most four and for all simple graphs of order $n$ having maximum degree at least $n-5$.
\end{abstract}

1991 Mathematics subject classification (Amer. Math. Soc.): 05 C 15.

Keywords and phrases. total colouring, total chromatic number.

\section{Introduction}

Throughout this paper, all graphs are finite, simple and undirected. Let $G$ be a graph. We denote its vertex set, edge set, complementary graph, chromatic index, the minimum degree of its vertices, and the maximum degree of its vertices by $V(G), E(G), \bar{G}, \chi_{1}(G), \delta(G)$, and $\Delta(G)$ respectively. A wellknown theorem of Vizing says that $\Delta(G) \leq \chi_{1}(G) \leq \Delta(G)+1$. A graph $G$ is said to be Class 1 (respectively Class 2) if $\chi_{1}(G)=\Delta(G)$ (respectively $\Delta(G)+$ 1). If $x \in V(G)$, we denote by $N_{G}(x)$ (or simply $N(x)$ ) the neighbourhood of $x$ and $d_{G}(x)$ (or simply $d(x)$ ) the degree of $x$. A vertex $x$ of $G$ is called a major vertex if $d(x)=\Delta(G)$. A vertex $y$ of $G$ is called a minor vertex if $y$ is not a major vertex. The subgraph of $G$ induced by all its major vertices is called the core of $G$ and is denoted by $G_{\Delta}$. If $F \subseteq E(G)$,

(C) 1992 Australian Mathematical Society 0263-6115/92 \$A2.00+0.00 
then $G-F$ is the graph obtained from $G$ by deleting $F$ from $G$. If $S \subseteq V(G)$, then $G[S]$ and $G-S$ denote the subgraphs of $G$ induced by $S$ and $V(G) \backslash S$ respectively. However, we write $G\left[v_{1}, v_{2}, \ldots, v_{k}\right]$ instead of $G\left[\left\{v_{1}, v_{2}, \ldots, v_{k}\right\}\right]$. We write $H \leq G$ if $H$ is an induced subgraph of $G$. A subset $S$ of vertices of $G$ is said to be independent (in $G$ ) if no two vertices of $S$ are adjacent in $G$. A graph $G$ is maximal if for any two nonadjacent vertices of $G$ at least one of them is a major vertex. The null graph of order $m$ and the complete graph of order $n$ are denoted by $O_{m}$ and $K_{n}$ respectively.

A total colouring $\pi$ of a graph $G$ is a mapping $\pi: V(G) \cup E(G) \rightarrow$ $\{1,2, \ldots\}$ such that

(i) no two adjacent vertices or edges have the same image;

(ii) the image of each vertex $x$ is distinct from the images of its incident edges.

The total chromatic number $\chi_{T}(G)$ of a graph $G$ is the smallest integer $k$ such that $G$ has a total colouring $\pi$ having image set $\{1,2, \ldots, k\}$.

From the definition of total chromatic number, it is clear that $\chi_{T}(G) \geq$ $\Delta(G)+1$. Behzad [1] and Vizing $[9,11]$ independently made the following conjecture.

Total Colouring Conjecture. For any graph $G, \chi_{T}(G) \leq \Delta(G)+2$.

This conjecture was proved for complete graphs by Behzad, Chartrand and Cooper [2]; for graphs $G$ having $\Delta(G) \leq 3$ by Rosenfeld [7] and Vijayaditya [8]; for graphs $G$ having $\Delta(G)=4$ by Kostochka [6]; for complete 3-partite graphs, complete balanced $r$-partite graphs by Rosenfeld [7]; for complete $r$ partite graphs by Yap [13]; for regular graphs of high degree by Chetwynd and Hilton [5]; and for graphs of order $n$ having $\Delta(G) \geq n-4$ by Yap, Wang and Zhang [14]. The main results of this paper are stated in the abstract above. The proof technique we use here is basically the same as that used in [14]. However, we introduce a few new techniques in settling some special cases when $n$ is small.

\section{Preliminary results}

We shall apply the following results.

THEOREM 2.1 (Rosenfeld [7], Vijayaditya [8] and Kostochka [6]). For any graph $G$ having $\Delta(G) \leq 4, \chi_{T}(G) \leq \Delta(G)+2$. 
Lemma 2.2. For any subgraph $H$ of a graph $G, \chi_{T}(H) \leq \chi_{T}(G)$.

Lemma 2.2 requires no proof. This lemma is often applied implicitly.

LemMA 2.3. If $G_{\Delta}$ is a forest, then $G$ is Class 1 .

Lemma 2.3 follows from some of Vizing's results (see for instance, [12, Theorem 3.3 and Corollary 3.6]). [14, Lemma 2.2] can be restated as follows.

LEMMA 2.4. Let $G$ be a graph of order $n$ and let $\Delta=\Delta(G)$. If $G$ contains an independent set $S$ where $|S| \geq n-\Delta-1$, then $\chi_{T}(G) \leq \Delta+2$.

Theorem 2.5 (Yap, Wang and Zhang [14]). For any graph $G$ of order $n$ having $\Delta(G) \geq n-4, \chi_{T}(G) \leq \Delta(G)+2$.

THEOREM 2.6 (Chetwynd and Hilton [3]). Let $G$ be a connected graph of order $n$ with three major vertices. Then $G$ is Class 2 if and only if each of the three major vertices is of degree $n-1$ and the remaining vertices have degree $n-2$.

THEOREM 2.7. Let $G$ be a bipartite graph with bipartition $(X, Y)$. Then $G$ contains a matching that saturates every vertex in $X$ if and only if $|N(S)| \geq$ $|S|$ for all $S \subseteq X$.

(A proof of Theorem 2.7 can be found in many textbooks on graph theory.)

THEOREM 2.8 (Chetwynd and Hilton [4]). Let $G$ be a connected graph having four major vertices. Then $G$ is Class 2 if and only if for some integer $n$, one of the following holds (for definition of valency-list, see [12]):

(i) the valency-list of $G$ is $(2 n-2)^{2 n-3}(2 n-1)^{4}$;

(ii) the valency-list of $G$ is $(2 n-2)(2 n-1)^{2 n-4}(2 n)^{4}$;

(iii) $G$ contains a bridge $e$ such that $G-e$ is the union of two graphs $G_{1}$ and $G_{2}$ where $\Delta\left(G_{1}\right) \leq 2 m-1$ for some integer $m<n$ and the valency-list of $G_{2}$ is either $(2 m-2)(2 m-1)^{2 m-4}(2 m)^{4}$ or $(2 m-1)^{2 m-2}(2 m)^{3}$.

\section{Proof of main results}

We shall now apply the above preliminary results to prove the main theorem (Theorem 3.2). We prove a general result first. 
LEMMA 3.1. Let $G$ be a graph of order $n$ having $\Delta(G)=n-k, k \geq 5$ and $n \geq 3 k-4$. If $G$ is maximal and $G \geq O_{k-2}$, then $\chi_{T}(G) \leq \Delta(G)+2$.

Proof. By Lemma 2.4, we can assume that $G \nsupseteq O_{k-1}$. Let $T=\left\{x_{1}, x_{2}\right.$, $\left.\ldots, x_{k-2}\right\}$ be a set of independent vertices in $G$. Let $V_{1}$ be the set of major vertices of $G$. Suppose $u, v \in V(G) \backslash T$ are not adjacent in $G$. Let $M_{1}$ be a matching in $H=G-T$ satisfying

(1) all the major vertices in $\{u, v\}$ are $M_{1}$-saturated;

(2) $\left|V\left(M_{1}\right) \cap V_{1}\right|$ is maximum among all the matchings in $H$ satisfying (1).

We now prove that $V_{1} \backslash T$ contains at most one $M_{1}$-unsaturated vertex. Suppose otherwise. Let $z_{1}, z_{2} \in V_{1} \backslash T$ be $M_{1}$-unsaturated. Since $\Delta(G)=$ $n-k, z_{1}$ is adjacent to at least $n-2 k+2$ vertices $a_{1}, a_{2}, \ldots, a_{n-2 k+2}$ in $H$. Clearly each $a_{i}$ is $M_{1}$-saturated. Let $b_{i} \in V(H)$ be such that $a_{i} b_{i} \in M_{1}, i \in I=\{1,2, \ldots, n-2 k+2\}$. Clearly $b_{i} \neq z_{2}$ for any $i \in I$ but $b_{i}$ can be $a_{j}$ for some $j \neq i$. Suppose $z_{2} b_{i} \in E(G)$ for some $i \in I$. Then replacing $a_{i} b_{i}$ in $M_{1}$ by $\left\{z_{1} a_{i}, z_{2} b_{i}\right\}$ we obtain a larger matching $M_{1}^{\prime}$ satisfying (1) but contradicting (2). Hence $z_{2} b_{i} \notin E(G)$, for all $i \in I$. (We call this argument the "Enlarge Matching Argument.") Since $z_{1}, b_{1}, b_{2}, \ldots, b_{n-2 k+2} \notin N\left(z_{2}\right)$, we have $n-k=d\left(z_{2}\right) \leq n-(n-2 k+$ $2+2)=2 k-4$. Hence $n=3 k-4$, and any other vertex of $G$ is adjacent to $z_{2}$. Let $A=\left\{a_{1}, a_{2}, \ldots, a_{n-2 k+2}\right\}, B=\left\{b_{1}, b_{2}, \ldots, b_{n-2 k+2}\right\}$. Suppose $A \neq B$. We first consider the case $A \cap B \neq \varnothing$. By symmetry, there are vertices $c_{i}, c_{j} \in N\left(z_{2}\right)$ such that $c_{i} c_{j} \in M_{1}$. Let $C$ be such a set of vertices. By the "Enlarge Matching Argument," each $b_{i} \in B \backslash A$ is not adjacent to any $b_{r} \in B$ and any $c_{s} \in C$. It is also not adjacent to $z_{1}$ and $z_{2}$. Hence $d\left(b_{i}\right) \leq(n-1)-(n-2 k+1)-4=2 k-6$. However replacing $a_{i} b_{i}$ in $M_{1}$ by $z_{1} a_{i}$ we have another matching $M_{1}^{\prime}$ satisfying conditions (1) and (2) and thus $b_{i} \in V_{1}$. Now $2 k-6 \geq d\left(b_{i}\right)=n-k$ contradicts the assumption that $n \geq 3 k-4$. Hence $A \cap B=\varnothing$. Now since $(n-2 k+2)+1 \geq k-1$ and $G\left[b_{1}, b_{2}, \ldots, b_{n-2 k+2}, z_{2}\right] \geq O_{k-1}$, we have another contradiction. Hence $V_{1} \backslash T$ contains at most one $M_{1}$-unsaturated vertex, say $z$.

Let $G_{1}=G-M_{1}, H_{1}=G_{1}-\{u, v\}$ and $V_{2}=\left\{h \in V\left(H_{1}\right) \mid d_{G_{1}}(h) \geq\right.$ $\Delta-1\}$. Since $G$ is maximal and $G[T]=O_{k-2}, T$ contains at most one minor vertex. Now let $Y=V(G) \backslash(T \cup\{u, v\})$. For any $S \subseteq T$ where $S$ consists of only major vertices, let $s=|S|$ and $r=|N(S) \cap Y|$. Then $s(n-k-2) \leq r(k-2)$ from which it follows that $r \geq s$. Hence by Theorem 2.7, $H_{1}$ contains a matching $M_{2}$ satisfying

(3) $z$ and all the major vertices in $T$ are $M_{2}$-saturated;

(4) $\left|V\left(M_{2}\right) \cap V_{2}\right|$ is maximum among all matchings in $H_{1}$ satisfying (3). We now show that $V_{2}$ contains at most two $M_{2}$-unsaturated vertices. Sup- 
pose otherwise. Let $w_{1}, w_{2}, w_{3} \in V_{2}$ be $M_{2}$-unsaturated. Then $w_{1}$ is adjacent to at least $n-k-3$ vertices $a_{1}, a_{2}, \ldots, a_{n-k-3}$ in $H_{1}$. Clearly each $a_{j}$ is $M_{2}$-saturated. Let $b_{j} \in V(G)$ be such that $a_{j} b_{j} \in M_{2}, j \in J=$ $\{1,2, \ldots, n-k-3\}$. By the "Enlarge Matching Argument," $w_{2} b_{j}, w_{3} b_{j} \notin$ $E\left(G_{1}\right)$ for all $j \in J$. Thus $n-k-1 \leq d_{G_{1}}\left(w_{i}\right) \leq n-(n-k-3+3)=k$, for each $i=2,3$. Since $n \geq 3 k-4$ and $k \geq 5$, we now have $k=5$ and $n=3 k-4=11$. Finally, for $n=11,5=d_{G_{1}}\left(w_{i}\right)(i=2,3)$ implies that $w_{i} \in N\left(a_{j}\right)$ for all $i=2,3, j=1,2,3$. If $b_{i} b_{j} \in E\left(G_{1}\right)$ for some $i \neq j$, then replacing $\left\{a_{i} b_{i}, a_{j} b_{j}\right\}$ in $M_{2}$ by $\left\{w_{1} a_{i}, b_{i} b_{j}, w_{2} a_{j}\right\}$ we obtain a larger matching $M_{2}^{\prime}$ satisfying (3) but contradicting (4). Hence $b_{i} b_{j} \notin E\left(G_{1}\right)$ for all $i, j$. Since $w_{i}, b_{i} \in V_{2}$ for all $i=1,2,3 \quad\left(b_{i} \in V_{2}\right.$ because we can replace $a_{i} b_{i}$ in $M_{2}$ by $\left.w_{1} a_{i}\right)$, it follows that $w_{i}, b_{i} \in N(u), N(v)$ for all $i=1,2,3$. Hence $d_{G_{1}}(u), d_{G_{1}}(v) \geq 6$ and so either $d(u) \geq 7$ or $d(v) \geq 7$ (because either $u$ or $v$ is a major vertex), which contradicts the fact that $\Delta(G)=11-5=6$. Hence $V_{2}$ contains at most two $M_{2}$-unsaturated vertices, say $w$ and $w^{\prime}$.

Now let $G^{*}$ be the graph obtained from $G_{2}=G_{1}-M_{2}$ by adding a new vertex $c^{*}$ and adding an edge joining $c^{*}$ to each vertex in $V(G) \backslash(T \cup\{u, v\})$. Then $\Delta\left(G^{*}\right)=n-k$ and $G^{*}$ has at most four major vertices. By Theorem 2.6 and Theorem 2.8, $\chi_{1}\left(G^{*}\right) \leq n-k$. Now any $(n-k)$-edge colouring $\pi$ can be modified to yield a total colouring $\varphi$ of $G$ using $(n-k)+2$ colours. Hence $\chi_{T}(G) \leq n-k+2$.

THEOREM 3.2. Let $G$ be a graph of order $n$ having $\Delta(G)=n-k$, where $k \geq 5$ and $n \geq \max (13,3 k-3)$. If $G$ is maximal and $G \geq O_{k-3}$, then $\chi_{T}(G) \leq \Delta(G)+2$.

Proof. Let $\Delta=\Delta(G)$. By Lemma 3.1, we also assume that $G \geq O_{k-2}$. Let $T=\left\{v_{1}, v_{2}, \ldots, v_{k-3}\right\}$ be a set of independent vertices in $G$. Let $V_{1}$ be the set of major vertices of $G$. Since $G \nsupseteq O_{k-2}$ and $\delta(\bar{G})=n-1-\Delta=k-$ 1 , we can find two pairs of nonadjacent vertices $\left\{x_{i}, y_{i}\right\}, i=1,2$, disjoint from $T$. Since $G$ is maximal, we may assume that $x_{1}, x_{2}, v_{1}, \ldots, v_{k-4}$ are major vertices. We first show that $H=G-T$ contains a matching $M_{1}$ satisfying

(5) all the major vertices in $\left\{x_{1}, y_{1}, x_{2}, y_{2}\right\}$ are $M_{1}$-saturated;

(6) $\left|V\left(M_{1}\right) \cap V_{1}\right|$ is maximum among all the matchings in $H$ satisfying (5).

We know that each major vertex of $G$ is of degree $n-k$. Hence each major vertex in $S=\left\{x_{1}, y_{1}, x_{2}, y_{2}\right\}$ is adjacent to at least $(n-k)-(k-3)-2=$ 
$n-2 k+1 \geq 4$ vertices in $V(G) \backslash(T \cup S)$. Hence, by Theorem $2.7, H$ contains a matching $M_{1}$ satisfying (5).

We now prove that $V_{1} \backslash T$ contains at most one $M_{1}$-unsaturated vertex. Suppose otherwise. Let $u_{1}, u_{2} \in V_{1} \backslash T$ be two $M_{1}$-unsaturated vertices. As in the proof of Lemma 3.1, $u_{1}$ is adjacent to at least $n-2 k+3$ vertices in $H$, whence $d\left(u_{2}\right) \leq n-(n-2 k+3+2)=2 k-5$, a contradiction. Hence $V_{1} \backslash T$ contains at most one $M_{1}$-unsaturated vertex, say $u$, in $H$.

Let $G_{1}=G-M_{1}, H_{1}=G_{1}-\left\{x_{1}, y_{1}\right\}$ and $V_{2}=\left\{h \in V\left(H_{1}\right) \mid d_{G_{1}}(h) \geq\right.$ $\Delta-1\}$. We now show that $H_{1}$ contains a matching $M_{2}$ satisfying

(7) $u$ and all the major vertices in $T \cup\left\{x_{2}, y_{2}\right\}$ are $M_{2}$-saturated;

(8) $\left|V\left(M_{2}\right) \cap V_{2}\right|$ is maximum among all the matchings in $H_{1}$ satisfying (7).

We know that $G\left[v_{1}, v_{2}, \ldots, v_{k-3}, x_{2}, y_{2}\right] \nsupseteq O_{k-2}$. Hence there exist integers $r \neq s$ such that $x_{2} v_{r}, y_{2} v_{s} \in E(G)$. Now each major vertex in $T \backslash\left\{v_{r}, v_{s}\right\}$ is adjacent to at least $n-k-4 \geq 2 k-7 \geq k-2$ vertices in $V(G) \backslash(T \cup S)$. By Theorem 2.7, there exists a matching $M_{2}^{\prime}$ that saturates all the major vertices in $T \backslash\left\{v_{r}, v_{s}\right\}$ in the bipartite graph having bipartition $T \backslash\left\{v_{r}, v_{s}\right\}$ and $V(G) \backslash(T \cup S)$. If $u$ is adjacent to some $v_{t} \in T \backslash\left\{v_{r}, v_{s}\right\}$ and $v_{t}$ is incident with an edge $e$ of $M_{2}^{\prime}$, we put $M_{2}=\left(M_{2}^{\prime} \backslash\{e\}\right) \cup\left\{u v_{t}\right\}$. Otherwise since $d(u)=\Delta$ and $\Delta-(k-5)-6 \geq k-4 \geq 1, u$ is adjacent to some $u^{\prime} \in V(G) \backslash\left(V\left(M_{2}^{\prime}\right) \cup S \cup\left\{v_{r}, v_{s}\right\}\right)$ and we can take $M_{2}=M_{2}^{\prime} \cup\left\{u u^{\prime}\right\}$.

We now show that $V_{2}$ contains at most one $M_{2}$-unsaturated vertex. Suppose otherwise. Let $z_{1}, z_{2} \in V_{2}$ be $M_{2}$-unsaturated. Now $z_{1}$ is adjacent to at least $\Delta-3$ vertices in $H_{1}$. Thus $\Delta-1 \leq d_{G_{1}}\left(z_{2}\right) \leq n-(\Delta-3+2)=k+1$, from which it follows that $n \leq 2 k+2$, a contradiction. Hence $V_{2}$ contains at most one $M_{2}$-unsaturated vertex, say $z$.

Let $G_{2}=G_{1}-M_{2}, H_{2}=G_{2}-\left\{x_{2}, y_{2}\right\}$ and $V_{3}=\left\{h \in V\left(H_{2}\right) \mid d_{G_{2}}(h) \geq\right.$ $\Delta-2\}$. We shall show that $H_{2}$ contains a matching $M_{3}$ satisfying

(9) every vertex in $\left\{v_{1}, v_{2}, \ldots, v_{k-3}, u, z, x_{1}, y_{1}\right\}$ whose degree in $G_{2}$ is $\Delta-1$ is $M_{3}$-saturated; (9).

(10) $\left|V\left(M_{3}\right) \cap V_{3}\right|$ is maximum among all the matchings in $H_{2}$ satisfying

We know that $G\left[v_{1}, v_{2}, \ldots, v_{k-3}, x_{1}, y_{1}\right] \nsupseteq O_{k-2}$. Hence there exist integers $r^{\prime} \neq s^{\prime}$ such that $x_{1} v_{r^{\prime}}, y_{1} v_{s^{\prime}} \in E(G)$. Now, each major vertex in $T \backslash\left\{v_{r^{\prime}}, v_{s^{\prime}}\right\}$ is adjacent to at least $(n-k-1)-4 \geq 2 k-8 \geq k-3$ vertices in $V(G) \backslash(T \cup S)$. Note that $d_{G_{2}}(u)=\Delta-1$ and $d_{G_{2}}(z)=\Delta-1$. Hence, as before and by Theorem 2.7, $\mathrm{H}_{2}$ contains a matching $M_{3}$ satisfying (9).

We next show that $V_{3}$ contains at most one $M_{3}$-unsaturated vertex. Suppose otherwise. Let $w_{1}, w_{2} \in V_{3}$ be $M_{3}$-unsaturated. As before, $w_{1}$ is adjacent to at least $\Delta-4$ vertices $a_{1}, a_{2}, \ldots, a_{\Delta-4}$ in $H_{2}$ and each $a_{i}$ is $M_{3}$ saturated. Let $b_{i} \in V\left(H_{2}\right)$ be such that $a_{i} b_{i} \in M_{3}, i=1,2, \ldots, \Delta-4$. By 
the "Enlarge Matching Argument," $b_{i} w_{2} \notin E\left(G_{2}\right)$ for all $i=1,2, \ldots, \Delta-$ 4. Thus $d_{G_{2}}\left(w_{2}\right) \leq n-(\Delta-4)-2=k+2$. Now $2 k-5 \leq n-k-2=$ $\Delta-2 \leq d_{G_{2}}\left(w_{2}\right) \leq k+2$ implies that $k \leq 7$ and $n \leq 2 k+4$.

We consider three cases separately.

Case 1. $b_{i} \notin A=\left\{a_{1}, a_{2}, \ldots, a_{\Delta-4}\right\}$ for $i=1,2, \ldots, \Delta-4$.

We first note that if $k=7$, then from the inequality $2 k-5 \leq \Delta-2 \leq$ $d_{G_{2}}\left(w_{2}\right) \leq k+2$, it follows that $d_{G_{2}}\left(w_{2}\right)=\Delta-2$ and thus $w_{2}$ is adjacent to every vertex in $A$; also if $k=6$ or $k=5$ (and thus $n \geq 13$ ), then $w_{2}$ is not adjacent to at most one vertex in $A$. Hence if $b_{i} b_{j} \in E\left(G_{2}\right)$ for some $1 \leq i<j \leq \Delta-4$, then replacing $\left\{a_{i} b_{i}, a_{j} b_{j}\right\}$ in $M_{3}$ by $\left\{w_{2} a_{i}, w_{1} a_{j}, b_{i} b_{j}\right\}$ (or $\left\{w_{2} a_{j}, w_{1} a_{i}, b_{i} b_{j}\right\}$ ), we obtain a larger matching $M_{3}^{\prime}$ satisfying (9) but contradicting (10). Hence $G_{2}\left[w_{1}, w_{2}, b_{1}, b_{2}, \ldots, b_{\Delta-4}\right]=O_{\Delta-2}$. Since $\left(M_{1} \cup M_{2}\right) \cap E\left(G\left[w_{1}, w_{2}, b_{1}, b_{2}, \ldots, b_{\Delta-4}\right]\right)$ is a union of paths and even cycles, and $\Delta-2=n-k-2 \geq 2 k-5$, we have $G\left[w_{1}, w_{2}, b_{1}, b_{2}, \ldots, b_{\Delta-4}\right] \geq$ $O_{k-2}$, a contradiction to the assumption.

Case 2. $b_{i} \in A$ and $b_{j} \notin A$ for some $1 \leq i, j \leq \Delta-4$.

Suppose $b_{1}, b_{2} \in A$, that is, $b_{1}=a_{2}$ and $b_{2}=a_{1}$, and suppose $b_{\Delta-4} \notin$ $A$. By symmetry, we also have $C=\left\{c_{1}, c_{2}, \ldots, c_{\Delta-r}\right\} \subseteq N_{H_{2}}\left(w_{2}\right),\left\{c_{1} c_{2}\right.$, $\left.c_{3} d_{3}, \ldots, c_{\Delta-4} d_{\Delta-4}\right\} \subseteq M_{3}$ and $d_{\Delta-4} \notin C$. First suppose $c_{\Delta-4}=a_{\Delta-4}$, say. Then $d_{\Delta-4}=b_{\Delta-4}$ and by the "Enlarge Matching Argument," $w_{1}, w_{2}$, $a_{1}, a_{2}, c_{1}, c_{2}, b_{3}, \ldots, b_{\Delta-5}, d_{3}, \ldots, d_{\Delta-5} \notin N_{H_{2}}\left(b_{\Delta-4}\right)$. (Note that $b_{i}$ may be equal to $d_{j}$ for some $i$ and $j$ such that $3 \leq i \leq j \leq \Delta-5$.) Hence

$$
2 k-5 \leq n-k-2=\Delta-2 \leq d_{G_{2}}\left(b_{\Delta-4}\right) \leq n-(\Delta-5)-5=k,
$$

from which it follows that $k=5$ and $n \leq 12$, a contradiction. Next, suppose $C \cap A=\varnothing$. Then the minimum order of $G$ is attained when $b_{\Delta-4}$ is the only vertex in $B=\left\{b_{1}, b_{2}, \ldots, b_{\Delta-4}\right\}$ such that $b_{\Delta-4} \notin A$. Thus $2(\Delta-5)+8 \leq n$ (note that $\left|\left\{x_{2}, y_{2}, w_{1}, w_{2}, a_{\Delta-4}, b_{\Delta-4}, c_{\Delta-4}, d_{\Delta-4}\right\}\right|=8$ ), from which it follows that $k=5$ and $n=12$, again a contradiction.

Case 3. $b_{j} \in A$ for $j=1,2, \ldots, \Delta-4$.

In this case $\Delta-4$ is even. We consider the following subcases separately.

(i) $k=7$. From $18=3 k-3 \leq n \leq 2 k+4=18$ we have $n=18$. However, we now also have $\Delta-4=n-k-4=n-11=7$ is odd, which is a contradiction.

(ii) $5 \leq k \leq 6$. By the "Enlarge Matching Argument," $d_{G_{2}}\left(a_{i}\right) \geq n-$ $k-2, i=1,2, \ldots, \Delta-4$, and every $a_{i}$ is not adjacent to any $v \in$ $V\left(G_{2}\right) \backslash\left(\left\{w_{1}, x_{2}, y_{2}\right\} \cup A\right)$. Hence $G_{2}\left[w_{1}, a_{2}, a_{2}, \ldots, a_{\Delta-4}\right]=K_{\Delta-3}$. Similarly, we have $G_{2}\left[w_{2}, c_{1}, c_{2}, \ldots, c_{\Delta-4}\right]=K_{\Delta-3}$. Thus each vertex in $W=$ $\left\{w_{1}, w_{2}\right\} \cup A \cup C$ must be adjacent to both $x_{2}$ and $y_{2}$, and consequently 
$2 \Delta-6 \leq d_{G_{2}}\left(x_{2}\right) \leq \Delta-2$ from which it follows that $\Delta \leq 4$, a contradiction. Hence $V_{3}$ has at most one $M_{3}$-unsaturated vertex.

Finally, let $G^{*}$ be obtained from $G_{3}=G_{2}-M_{3}$ by adding a new vertex $c^{*}$ and adding an edge joining $c^{*}$ to each vertex in $V\left(G_{3}\right) \backslash\left(T \cup\left\{x_{1}, x_{2}, y_{1}, y_{2}\right\}\right)$. Then $\Delta\left(G^{*}\right)=\Delta-1$, and by the choices of $M_{1}, M_{2}$ and $M_{3}, G^{*}$ has at most four major vertices. Hence, by Theorem 2.6 and Theorem $2.8, \chi_{1}\left(G^{*}\right)=\Delta-1$ and if $\pi$ is a $(\Delta-1)$-edge colouring of $G^{*}$, then we can modify $\pi$ to a total colouring $\varphi$ of $G$ using $(\Delta-1)+3$ colours. Hence $\chi_{T}(G) \leq(\Delta-1)+3=$ $\Delta+2$.

The proof of Theorem 3.2 is complete.

We shall also require the following lemmas.

LEMMA 3.3. Let $G$ be a graph of order 10 having $\Delta(G)=5$. If $G$ is maximal and $G \geq O_{3}$, then $\chi_{T}(G) \leq \Delta(G)+2$.

Proof. The proof is almost identical to the proof of Lemma 3.1. Hence we need only to modify some parts of the proof of Lemma 3.1.

In the case of showing that $V_{1}$ contains at most one $M_{1}$-unsaturated vertex, we let $\{x\}=V(G) \backslash\left(T \cup\left\{z_{1}, z_{2}, a_{1}, a_{2}, b_{1}, b_{2}\right\}\right)$ if $b_{i} \notin A=$ $\left\{a_{1}, a_{2}\right\}$. Then clearly $x z_{2} \notin E(G)$. Hence $a_{1}, a_{2} \in N\left(z_{2}\right)$. Also since $G\left[b_{1}, b_{2}, z_{1}, z_{2}\right] \neq O_{4}$, we have $b_{1} b_{2} \in E(G)$. Consequently, by the "Enlarge Matching Argument," $a_{1}, a_{2} \notin N\left(z_{2}\right)$, a contradiction. On the other hand, if $b_{i} \in A$, we let $c_{1}, c_{2} \in N_{H}\left(z_{2}\right)$. Then by the "Enlarge Matching Argument," $a_{1}, a_{2}, c_{1}, c_{2}, z_{1}$ and $z_{2}$ are all adjacent to each vertex in $T$, which is false. Hence $V_{1}$ contains at most one $M_{1}$-unsaturated vertex, $z$ say.

In the case of showing that $V_{2}$ contains at most two $M_{2}$-unsaturated vertices, we now have $n-k-1 \leq d_{G_{1}}\left(w_{i}\right) \leq k$ for each $i=2,3$.

Again, we let $\{x\}=V(G) \backslash\left\{u, v, w_{1}, w_{2}, w_{3}, a_{1}, a_{2}, b_{1}, b_{2}\right\}$. Then $x$ is $M_{2}$-unsaturated and $x w_{i} \notin E\left(G_{1}\right)$ for all $i=1,2,3$. By the "Enlarge Matching Argument," $b_{1}, b_{2} \in V_{2}$, and $a_{1}, a_{2} \in N_{H}\left(w_{j}\right), j=2,3$. Hence $x b_{1}, x b_{2}, b_{1} b_{2} \notin E\left(G_{1}\right)$. Thus $b_{1}, b_{2}, w_{1}, w_{2}, w_{3} \in N(u) \cap N(v)$ and consequently either $d(u) \geq 6$ or $d(v) \geq 6$, a contradiction.

LEMMA 3.4. Let $G$ be a graph of order $n=10,11$ or 12 . Suppose $\Delta(G)=n-5, G$ is maximal and $G \nsupseteq O_{3}$. Then $\chi_{T}(G) \leq \Delta(G)+2$.

Proof. We shall apply the technique used in the proof of Theorem 3.2 by explicitly constructing out the three matchings $M_{1}, M_{2}$ and $M_{3}$. 
Let $u_{1} \in V(G)$ be such that $d\left(u_{1}\right)=\delta=\delta(G)$ and let $v \in V(G)$ be such that $u_{1} v \notin E(G)$. Thus $v$ is a major vertex. Let $N_{\bar{G}}(v)=\left\{u_{1}, u_{2}, u_{3}, u_{4}\right\}$ and $N_{G}(v)=\left\{v_{1}, v_{2}, \ldots, v_{n-5}\right\}$. Since $G \geq O_{3}, G\left[u_{1}, u_{2}, u_{3}, u_{4}\right]=K_{4}$. If $d\left(u_{1}\right)=3$, then $N_{\bar{G}}\left(u_{1}\right)=\left\{v, v_{1}, v_{2}, \ldots, v_{n-5}\right\}$ forms a clique in $G$, that is, $G=K_{4} \cup K_{n-4}$. Thus $\chi_{T}(G) \leq \Delta(G)+2$. Hence we assume that $d\left(u_{1}\right)=\delta \geq 4$. Let $v_{1} \in N\left(u_{1}\right)$ and let $v_{2}$ be such that $v_{1} v_{2} \notin E(G)$. Since $\delta \geq 4$, we may also assume that $v_{n-6}, v_{n-5} \notin N\left(u_{1}\right)$ (note that there is also another vertex $v_{j}, 2 \leq j \leq n-7$, such that $v_{j} \notin N\left(u_{1}\right)$ but we shall not specifically assume which one.) Thus $G\left[v, v_{n-6}, v_{n-5}\right]=K_{3}$.

We now consider the following cases separately.

Case 1. $n=10$ or 11 .

(i) Suppose $v_{3} \notin N\left(v_{n-6}\right) \cap N\left(v_{n-5}\right)$, say $v_{3} \notin N\left(v_{n-6}\right)$.

For $n=10, G-\left\{v_{1}, v_{2}\right\}$ has a 1-factor $M_{1}=\left\{u_{1} u_{2}, u_{3} u_{4}, v_{3} v, v_{4} v_{5}\right\}$, $G-M_{1}-\left\{v_{3}, v_{4}\right\}$ has a 1 -factor $M_{2}=\left\{u_{1} u_{4}, u_{2} u_{3}\right\} \cup F G\left[v_{1}, v_{2}, v, v_{5}\right]$ where $F G\left[v_{1}, v_{2}, v, v_{5}\right]$ denotes a 1-factor in $G\left[v_{1}, v_{2}, v, v_{5}\right]$, and we can choose $M_{3}$ to be any maximum matching in $G-\left(M_{1} \cup M_{2}\right)-\left\{u_{1}, v\right\}$.

For $n=11$, we have $G\left[v_{4}, v_{1}, v_{2}\right] \neq O_{3}, G\left[v_{4}, v_{3}, v_{5}\right] \neq O_{3}$ and $G\left[v_{4}, u_{1}, v_{6}\right] \neq O_{3}$. Hence by symmetry, we can assume that $v_{4} u_{4} \notin$ $E(G)$. Now $G-\left\{v_{1}, v_{2}\right\}$ has a matching $M_{1}=\left\{v_{3} u_{1}, v_{4} v, v_{5} v_{6}, u_{3} u_{4}\right\}$ which misses $u_{2}, G-M_{1}-\left\{v_{3}, v_{5}\right\}$ has a matching $M_{2}=\left\{u_{1} u_{4}, u_{2} u_{3}\right\} \cup$ $F G\left[v, v_{1}, v_{2}, v_{4}\right]$ which misses $v_{6}$, and $G-\left(M_{1} \cup M_{2}\right)-\left\{u_{4}, v_{4}\right\}$ has a matching $M_{3}=\left\{u_{1} u_{2}, v v_{6}\right\} \cup F G\left[v_{1}, v_{2}, v_{3}, v_{5}\right]$ which misses $u_{3}$.

(ii) $v_{3} v_{n-6}, v_{3} v_{n-5} \in E(G)$.

By symmetry, we can also assume that $v_{4} v_{n-6}, v_{4} v_{n-5} \in E(G)$ if $n=11$. Thus, by symmetry again, we may further assume that $v_{n-6} u_{3}, v_{n-5} u_{4} \notin$ $E(G)$.

For $n=10, G-\left\{v_{1}, v_{2}\right\}$ has a 1-factor $M_{1}=\left\{u_{1} u_{2}, u_{3} u_{4}, v_{3} v, v_{4} v_{5}\right\}$, $G-M_{1}-\left\{u_{1}, v_{5}\right\}$ has a 1-factor $M_{2}=\left\{v_{3} v_{4}, u_{2} u_{4}\right\} \cup F G\left[v_{1}, v_{2}, v, u_{3}\right]$, and we can choose $M_{3}$ to be any maximum matching in $G-\left(M_{1} \cup M_{2}\right)-\left\{u_{4}, v\right\}$.

For $n=11, G-\left\{v_{1}, v_{2}\right\}$ has a matching $M_{1}=\left\{u_{1} u_{4}, u_{2} u_{3}, v_{3} v, v_{5} v_{6}\right\}$ which misses $v_{4}, G-M_{1}=\left\{u_{3}, v_{5}\right\}$ has a matching $M_{2}=\left\{u_{2} u_{4}, v_{3} v_{6}\right\} \cup$ $F G\left[v_{1}, v_{2}, v_{4}, v\right]$ which misses $u_{1}$, and $G-\left(M_{1} \cup M_{2}\right)-\left\{u_{4}, v_{6}\right\}$ has a matching $M_{3}=\left\{v_{4} v, u_{1} u_{2}\right\} \cup F G\left[v_{1}, v_{2}, v_{5}, u_{3}\right]$ which misses $v_{3}$.

Case 2. $n=12$.

This case is very similar to Case 1 . Although the length of proof of Case 2 is slightly shorter than that of Case 1 , the proof is very technical and thus we omit it.

Finally, from the above results we deduce

THEOREM 3.5. For any graph $G$ of order $n$ having $\Delta(G)=n-5$,

$$
\chi_{T}(G) \leq \Delta(G)+2 \text {. }
$$


Proof. By Lemma 2.2, we can assume that $G$ is maximal. By Lemma 3.1 , if $n \geq 11$ and $G \geq O_{3}$, then this theorem is true. By Theorem 3.2, if $n \geq 13$, then this theorem is also true. By Theorem 2.1, this theorem is true for $n \leq 9$. Hence we need only to consider the following remaining cases:

(i) $n=10$ and $G \geq O_{3}$;

(ii) $n=10,11$ or 12 and $G \nsupseteq O_{3}$.

However, these two cases have been settled by Lemma 3.3 and Lemma 3.4.

Remark. From the proofs of Theorem 3.2 and Theorem 3.5, we see that the technique we apply will be extremely difficult to prove that the TCC also holds for graphs of order $n$ having $\Delta(G)=n-6$.

ACKNOWLEDGMENT. We thank the referee for valuable comments.

\section{References}

[1] M. Behzad, Graphs and their chromatic numbers (Doctoral Thesis Michigan State Univ., 1965).

[2] M. Behzad, G. Chartrand and J. K. Cooper, 'The colour numbers of complete graphs', J. London Math. Soc. 42 (1967), 226-228.

[3] A. G. Chetwynd and A. J. W. Hilton, 'Regular graphs of high degree are 1-factorizable', Proc. London Math. Soc. 50 (3) (1985), 193-206.

[4] A. G. Chetwynd and A. J. W. Hilton, 'The chromatic index of graphs with at most four vertices of maximum degree' in: Proc. of the Fifteenth Southeastern Conf. on Combinatorics, Graph Theory and Computing (Baton Rouge, La., 1984) Congr. Numer. 43 (1984), 221-248.

[5] A. J. W. Hilton, 'Recent results on edge-colouring graphs, with applications to the totalchromatic number', J. Combin. Math. and Combin. Computing 3 (1988), 121-134.

[6] A. V. Kostochka, 'The total coloring of a multigraph with maximal degree 4', Discrete Math. 17 (1977), 161-163.

[7] M. Rosenfeld, 'On the total colouring of certain graphs', Israel J. Math. 9 (3) (1971), 396-402.

[8] N. Vijayaditya, 'On total chromatic number of a graph', J. London Math. Soc. (2) 3 (1971), 405-408.

[9] V. G. Vizing, 'On an estimate of the chromatic class of a p-graph' (Russian), Diskret. Analiz 3 (1964), 25-30.

[10] V. G. Vizing, 'Critical graphs with a given chromatic class' (Russian), Diskret. Analiz 5 (1965), 9-17.

[11] V. G. Vizing, 'Some unsolved problems in graph theory', Uspehi Mat. Nauk 23 (1968), 117-134 (Russian Math. Surveys 23 (1968), 125-142).

[12] H. P. Yap, Some topics in graph theory (Cambridge Univ. Press, 1986).

[13] H. P. Yap, 'Total colourings of graphs', Bull. London Math. Soc. 21 (1989), 159-163.

[14] H. P. Yap, Wang Jian-Fang and Zhang Zhongfu, 'Total chromatic number of graphs of high degree', J. Austral. Math. Soc. Ser. A 47 (1989), 445-452.

National University of Singapore

10 Kent Ridge Crescent

Singapore 0511 